\title{
TEMPERATURE DEPENDENCE OF RAMAN SCATTERING BY MAGNONS IN BULK-LIKE MBE-GROWN MnTe FILMS*
}

\author{
W. SzuszkieWicz ${ }^{a}$, J.F. Mohrange ${ }^{b}$, M. Jouanne ${ }^{b}$, M.A. Kanehisa ${ }^{b}$, \\ R. SWIRKowICz ${ }^{c}$, E. DYNoWSKA ${ }^{a}$, E. J ANIK $^{a}$, T. WOJTOWICZ ${ }^{a}$ \\ AND J. KossuT ${ }^{a}$ \\ ${ }^{a}$ Institute of Physics, Polish Academy of Sciences \\ Al. Lotników 32/46, 02-668 Warszawa, Poland \\ ${ }^{b}$ Laboratoire de Milieux Désordonnés et Hétérogènes, UPMC \\ Case 86, 4 place Jussieu, 75252 Paris Cédex 05, France \\ ${ }^{c}$ Warsaw Technical University, Koszykowa 75, 00-662 Warszawa, Poland
}

Temperature dependence of the magnon frequency was studied for cubic MnTe epilayers by the Raman scattering measurements. Experimental data are compared to the results of theoretical calculations performed within the framework of the Heisenberg model using Green's function formalism.

PACS numbers: 75.30.Ds, 75.50.Pp, 78.30.-j

Zinc-blende MnTe is an interesting magnetic semiconductor due to its unusual magnetic properties (all zinc-blende $\mathrm{Mn}$ chalcogenides are unique examples of fcc Heisenberg antiferromagnets with dominant nearest-neighbor interaction). Below the Néel temperature $T_{\mathrm{N}}(\approx 70 \mathrm{~K})$ cubic MnTe possesses the antiferromagnetic order of AFIII type [1]. As it was previously shown, the Raman scattering is an effective experimental method to study collective magnetic excitations (spin waves, magnons) in this material [2,3].

The goal of this work was to investigate both experimentally and theoretically the temperature dependence of such spin excitations. Preliminary experimental results have already been presented [3]. In this paper the Raman scattering by magnons was studied with a much better spectral resolution than previously. We also extended the measurements to the whole temperature range below $T_{\mathrm{N}}$. Moreover, we present the results of the first attempt to describe theoretically the temperature dependence of the magnon frequency in MnTe within the framework of the Heisenberg model.

MnTe films of zinc-blende structure were grown by molecular beam epitaxy (MBE) on semi-insulating (001) GaAs substrates. A thin (usually $1.5 \mathrm{~nm}$ thick)

${ }^{*}$ This work is supported in part by PB750/T08/95/09 grant from the Committee for Scientific Research (Poland). 
ZnTe layer was employed in order to reduce a strong mismatch between a 2-4 $\mu \mathrm{m}$ thick CdTe buffer layer and GaAs substrate and to stabilize the growth in the [001] direction. The final MnTe layer thickness was of the order of 3-5 micrometers. Quality of MnTe layers was assessed by X-ray diffraction using Co $K_{\alpha}$ radiation. A typical full-width at half maximum of the rocking curves was of the order of 12 arcmin, no inclusions of the other phases (e.g., hexagonal MnTe or Te) were detected.

Raman scattering experiments were performed in a quasi backscattering geometry using a Jobin Yvon U1000 double spectrometer equipped with holographic grating and a S20 photomultiplier. For the excitation we selected several $\mathrm{Ar}^{+}$and $\mathrm{Kr}^{+}$laser lines at a power of the order of $150 \mathrm{~mW}$ focussed to a $100 \mu \mathrm{m}$ diameter spot. The samples were mounted on the cold finger of a continuous-flow helium cryostat, the temperature was determined using a temperature gauge pasted just behind the samples. Raman scattering spectra were recorded with a spectral resolution close to $1 \mathrm{~cm}^{-1}$.

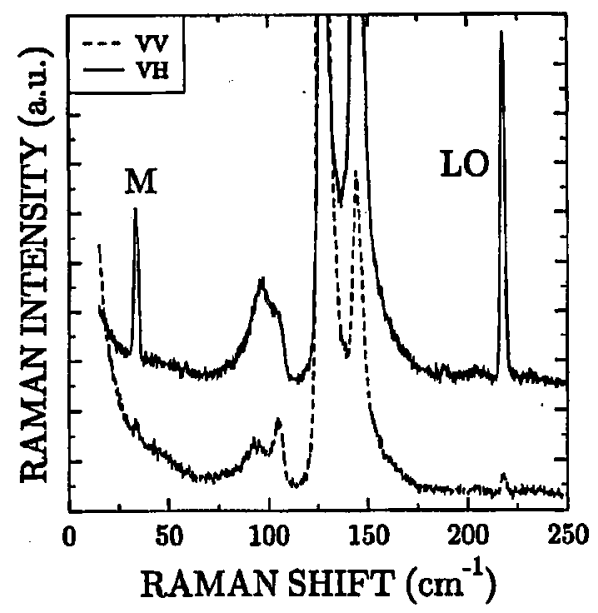

Fig. 1. Raman scattering spectrum taken for MnTe epilayer at $27 \mathrm{~K}$. Upper curve corresponds to $\bar{z}(x, y) z$ polarization (for which the Raman scattering by magnons is allowed due to the selection rules), the lower curve was obtained in the $\bar{z}(x, x) z$ polarization (scattering by magnons forbidden).

The low-frequency part of the typical Raman spectra in the $\bar{z}(x, y) z$ and $\bar{z}(x, x) z$ polarizations taken in MnTe sample at a low temperature are shown in Fig. 1. A peak (well-pronounced at $34 \mathrm{~cm}^{-1}$ in the $\bar{z}(x, y) z$ polarization) was observed at all temperatures below $T_{N}$ (Fig. 2), its intensity decreases with increasing temperature until it disappears above $T_{\mathrm{N}}$. The polarization and the temperature behavior of this peak as well as its frequency (close to the value expected on the basis of the Raman scattering by magnons, investigated in bulk CdMnTe mixed crystals $[4,5])$, demonstrated that it is due to the Raman scattering by one-magnon excitations (as previously discussed in Refs. [2,3]). The abrupt disappearance of 


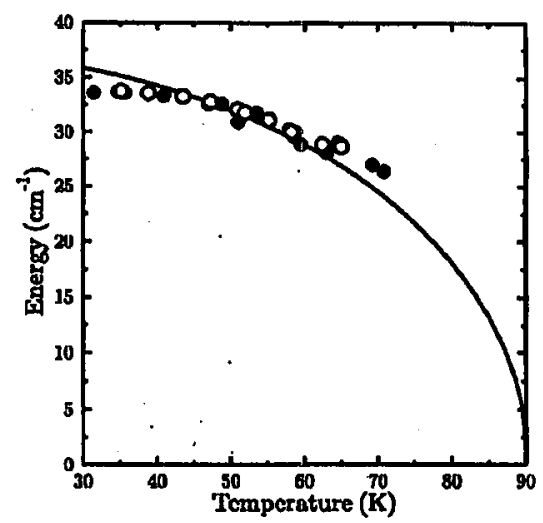

Fig. 2. Temperature dependence of the magnon frequency in two MnTe samples. Points correspond to the experimental data, solid line is the result of the theoretical calculations (see details in text). The temperature values were determined with the accuracy of a few $\mathrm{K}$ (e.g., at $T \approx 70 \mathrm{~K}$ this accuracy is $3 \mathrm{~K}$ ).

the magnon peak at $T_{\mathrm{N}}$ in the Raman experiment seems to be consistent with very sharp changes in the sublattice magnetization in this temperature region observed in neutron scattering measurements [1]. According to Ref. [1] such strong changes can suggest the first-order magnetic phase transition in MnTe. The full-width at half-maximum (FWHM) of the single-magnon line, which is below $1 \mathrm{~cm}^{-1}$ at a low temperature, increases with temperature and reaches a value of about $3 \mathrm{~cm}^{-1}$ at a temperature close to the magnetic phase transition temperature (where magnon frequency is still above $25 \mathrm{~cm}^{-1}$ ). At the lowest temperatures studied the FWHM saturates indicating that it is limited by the spectral resolution of the apparatus. In other words, the above-mentioned results demonstrate that magnon damping is quite small and the spin waves are well-defined excitations in the entire temperature range corresponding to the antiferromagnetic order in $\mathrm{MnTe}$.

To characterize the temperature dependence of the one-magnon line, the spectra were numerically fitted by the least squares method to a linear response function of a damped oscillator. The broad band under the magnon peak was modelled by a constant background. For each peak three parameters were adjusted (the frequency $\omega$, damping $\Gamma$ and the intensity $A$ ). Whenever possible, both Stokes and anti-Stokes parts of the spectra were independently fitted increasing the accuracy of determination of $\omega$ and $\Gamma$. Experimental curves obtained for the temperature dependence of the magnon energy were compared to those found on the basis of theoretical approach. As mentioned previously, neutron diffraction studies established existence of a long-range antiferromagnetic order of type III in MnTe epilayers [1]. The systems can be well described within the framework of the Heisenberg model with exchange interactions $J_{1}$ and $J_{2}$ for the nearest and the next-nearest neighbors, respectively, taken into account. To account for the observation of the one-magnon peak in the Raman spectra, it is mandatory to include the magnetic anisotropy in the model. This anisotropy energy was taken in the 
form: $\omega_{\mathrm{A}}=C\left\langle S^{z}\right\rangle / S$, where $C$ is a constant treated as an adjustable parameter and $\left\langle S^{z}\right\rangle$ corresponds to the magnetic moment of the sublattice at a temperature $T$. It should be stressed that MnTe films several micrometers thick were used so the surface effects had practically no influence on the observed spectra. Therefore, the results obtained for these bulk-like layers can be interpreted within the framework of the theory describing the magnetic properties of bulk materials. (The situation could be quite different if one attempts to analyze much thinner MnTe slabs. For a 1-micrometer thick MnTe layer the strain effects strongly affect the magnetic domain structure, as it was demonstrated in Ref. [1].) Spin-wave energy calculated for wave vector $q=0$ in the case of type III antiferromagnet can be given in the following form: $E_{\omega}=\left[\omega_{\mathrm{A}}\left(\omega_{\mathrm{A}}+2 \omega_{\mathrm{E}}\right)\right]^{1 / 2}$, where $\omega_{\mathrm{A}}$ corresponds to anisotropy energy and $\omega_{\mathrm{E}}=2 J\left\langle S^{z}\right\rangle$ with $J=8 J_{1}+2 J_{2}$ represents the exchange energy. Magnon-magnon interactions are not taken into account in the formula describing $E_{\omega}$. The spin-wave energy in such an approach depends on temperature only through the spontaneous magnetization $\langle S(T)\rangle$. This dependence can be calculated within Green's function formalism [6]. The theoretical calculations were performed for the following parameter values: $J_{1}=6.7 \mathrm{~K}, J_{2}=0.1 J_{1}, C=5.9 \mathrm{~K}$. The first two values are taken from Ref. [1] (the value of $J_{1}$ used here is the upper limit deduced from the neutron diffraction data in the bulk CdMnTe mixed crystals). The anisotropy value was obtained from our low-temperature Raman scattering data. In consistency with neutron diffraction measurements, the anisotropy field is very weak in comparison to the exchange field [1]. It should be mentioned that in our previous paper [3], the temperature dependence of the magnon frequency was described within the molecular field approach with the exchange integrals used for these materials in Ref. [4]. However, in such an approach the anisotropy field (or energy) is significantly overestimated. The results of the present calculations are shown in Fig. 2. The best fit is obtained in the middle temperature range. There are some discrepancies for the lowest and the highest temperatures. It is possible. that in these region systematic error appears due to the accuracy of fit. Moreover, the magnon-magnon interaction, probably, cannot be entirely neglected in the whole temperature range below $T_{\mathrm{N}}$.

\section{References}

[1] T.M. Giebułtowicz, P.K. Kłosowski, N. Samarth, H. Luo, J.K. Furdyna, J.J. Rhyne, Phys. Rev. B 48, 12817 (1993).

[2] W. Szuszkiewicz, M. Jouanne, E. Dynowska, E. Janik, G. Karczewski, T. Wojtowicz, J. Kossut, Acta Phys. Pol. A 88, 941 (1995).

[3] W. Szuszkiewicz, E. Dynowska, E. Janik, G. Karczewski, T. Wojtowicz, J. Kossut, M. Jouanne, W. Gębicki, in: Proc. 23 ICPS, Berlin 1996, Eds. M. Scheffler, R. Zimmermann, Vol. 1, World Scientific, Singapore 1996, p. 385.

[4] S. Venugopalan, A. Petrou, R.R. Gałazka, A.K. Ramdas, Solid State Commun. 38, 365 (1981); S. Venugopalan, A. Petrou, R.R. Gałazka, A.K. Ramdas, S. Rodriguez, Phys. Rev. B 25, 2681 (1982).

[5] M. Grynberg, M. Picquart, J. Phys. C Solid State Phys. 14, 4677 (1981); M. Picquart, M. Grynberg, J. Appl. Phys. 53, 8166 (1982).

[6] R. Świrkowicz, to be published in J. Phys. Condens. Malter. 